for 4 weeks or 12 weeks. Children were followed up at 1,3 , and 6 months of illness. The primary outcome was proportion of children developing seizure recurrence over 6 months follow up. The secondary outcome was to study factor(s) associated with seizure recurrence.

Results Out of 232 children with Acute Encephalitis Syndrome, 60 children were found to be eligible for randomization in two groups. Baseline demographics were comparable (except duration of illness) between the groups. None of the children developed any seizure recurrences in the follow up period. Although, 8 children had neurological deficits and 9 children had EEG abnormality, seizure recurrences were not seen in any of these children.

Conclusions The present study suggests that a shorter duration (4 weeks) of anti-epileptic drug therapy is comparable with 12 weeks anti-epileptic drugs for preventing seizure recurrences over a six-month follow-up period in this cohort of children with Acute Encephalitis Syndrome.

The trial was registered with Clinical Trial Registry of India (CTRI/2017/06/008783) and Clinicaltrial.gov (NCT03181945).

\section{G303 ACUTE CLINICAL EMERGENCIES ON THE PAEDIATRIC NEUROSCIENCES WARD: CAN WE IMPROVE PREDICTION AND REDUCE RISK?}

${ }^{1}$ MR Eyre, ${ }^{2} \mathrm{~L}$ Andre, ${ }^{1} \mathrm{R}$ Robinson. ${ }^{1}$ Paediatric Neurology Department, Great Ormond Street Hospital for Children NHS Foundation Trust, London, UK; ${ }^{2}$ Resuscitation Department, Great Ormond Street Hospital for Children NHS Foundation Trust, London, UK

\subsection{6/archdischild-2018-rcpch.295}

Background Paediatric inpatients with complex neurological problems can rapidly deteriorate and arrest on the ward. Various early warning risk-scoring systems are used, but none apply specifically to this patient cohort. The aim of this study was to identify modifiable risk factors and optimise patient safety.

Methods Our tertiary neurosciences unit comprises 24 acute beds (8 HDU) serving children with complex neurological, neurosurgical and craniofacial disorders. All calls to the clinical emergency team (CET) were prospectively audited.

Results Over 10.2 years the CET responded to 128 calls in 98 children (median age $2.8 \mathrm{y}$, range $2 \mathrm{~d}-19 \mathrm{y}, 52 \%$ female). Diagnoses included epilepsy (37\%), hydrocephalus (31\%), CNS tumour (14\%), craniofacial disorders (8\%) and epilepsy surgery (4\%). $19 \%$ of events followed recent surgery or general anaesthetic. Most recent Children's Early Warning Score (CEWS) was median 1 (IQR 0-3) at median 56 min pre-event (IQR 29-110).

Events included respiratory arrest $(88 \%)$ and cardiac arrest (4.7\%). Utstein-style categorization was used to classify the primary cause: $72 \%$ neurological, including seizure-related apnoea in $41 \%$ and raised intracranial pressure in $25 \%$ (hydrocephalus 16\%, acute haemorrhage 5.5\%); 22\% respiratory, including central or obstructive apnoea in $9.4 \%$, benzodiazepine-related apnoea in $6.3 \%$, and blocked tracheostomy in $4.7 \% ; 3.1 \%$ circulatory (septic shock, hypovolaemia). 33\% of events were regarded as potentially preventable by the attending CET.

In addition to basic supportive care, interventions on the ward included endotracheal intubation in 16\%, mannitol/ hypertonic saline in $9.4 \%$ and adrenaline in $2.3 \%$. No shockable rhythms were identified. One death occurred during resuscitation and $33 \%$ of survivors were transferred to ICU; all were alive at 24 hours. In 11 consecutive patients prospectively followed up, all survived to discharge at median 11 days (range 3-93) post-event; 86\% were alive at 1 year.

Conclusions Respiratory arrests secondary to epileptic seizure, raised intracranial pressure, central or obstructive apnoea and benzodiazepine administration are potentially predictable. CEWS had poor sensitivity for predicting imminent deterioration in paediatric neurosciences patients. Analysis of pre-event clinical observations in cases and controls will enable development of a deterioration-prediction model specific to this patient cohort.

\section{G304 DELPHI CONSENSUS PROCESS FOR THE UK GUIDELINES FOR MANAGEMENT AND SURVEILLANCE OF TUBEROUS SCLEROSIS COMPLEX}

${ }^{1} \mathrm{~S}$ Amin, ${ }^{2} \mathrm{C}$ Kingswood, ${ }^{3} \mathrm{~F}$ O'Callaghan. 'University Hospitals Bristol, Bristol, UK; ${ }^{2}$ Royal Brighton Hospital, Brighton, UK; ${ }^{3}$ University College London, London, UK

\subsection{6/archdischild-2018-rcpch.296}

Background Tuberous Sclerosis Complex (TSC) is a multi-systemic genetic disease. The severity of TSC can vary among affected individuals. Complications of TSC can be life threatening, with significant impact on patients' quality of life. Management may vary dependent on treating physician, local and national policies and funding. There are no current UK guidelines. We conducted a Delphi consensus process to gain consensus on the management of patients with TSC in the UK.

Methods We invited 86 clinicians and researchers to complete an online survey in two rounds. All the people surveyed were based in the UK. Clinicians were identified through the regional TSC clinics, and researchers were identified through publications. In round one, 55 questions were asked. They were related to surveillance and management recommendations for those newly diagnosed, suspected, or already diagnosed with TSC. In round two, 18 questions were asked to obtain consensus on the outstanding points that had been contentious in round one or needed clarification.

Results 51 (60\%) responded to the survey. Three rounds were required to achieve consensus. The responders were neurologists, nephrologists, psychiatrists, psychologists, oncologists, general paediatricians, dermatologists, urologists, radiologists, geneticists, neurosurgeons, pulmonologists and neurodisability clinicians. A priori consensus was defined as $70 \%$ agreement among participants. The Delphi process is now complete and the consensus management recommendations will be presented at conference.

Conclusions This new UK guideline for the management and surveillance of TSC patients provides a realistic, cost effective, an evidence-based approach for best clinical care delivered for individuals with tuberous sclerosis complex in the UK.

\section{G305 HOW MUCH VARIATION IN PAEDIATRIC EPILEPSY ADMISSION RATES IN ENGLAND CAN BE EXPLAINED BY VARIATION IN UNIT-LEVEL PERFORMANCE IN THE NATIONAL CLINICAL AUDIT (EPILEPSY12)}

${ }^{1} S$ Arora, ${ }^{1} \mathrm{C}$ Sherlaw-Johnson, ${ }^{2} \mathrm{CR}$ Cheung, ${ }^{3} \mathrm{DS}$ Hargreaves. ${ }^{1}$ Research Department, The Nuffield Trust, London, UK; ${ }^{2}$ Department of General Paediatrics, Evelina London Children's Hospital, London, UK; ${ }^{3}$ Population, Policy and Practice Programme, UCL Great Ormond Street Institute of Child Health, London, UK

10.1136/archdischild-2018-rcpch.297 
Background The UK has higher rates of epilepsy deaths among children and young people than comparable countries, and poor care quality is a contributing factor a significant proportion of epilepsy-related deaths. Admission rates are widely used as a proxy measure of care quality in other paediatric conditions. For epilepsy, admission rates vary 4-5 fold between areas in England and the national clinical audit of paediatric epilepsy care (Epilepsy 12) shows wide variation in measures of care quality. It is not known whether better audit performance is associated with lower admission rates.

Objective To investigate the degree to which better performance in the national clinical audit is associated with lower admission rates for children and young people (0-19 years) with epilepsy.

Methods Independent variables: 12 unit-level quality indicators including access to paediatric, specialist nurse and paediatric neurologist expertise.(Round 1=2009-12; Round 2=2014) $\mathrm{n}=144$ units.

Dependent variable: unit-level epilepsy admission rate (admissions calculated from Hospital Episode Statistics (HES 2011/ 12-2013/14); estimated unit catchment populations derived from age-specific, all-cause admissions and population data).

Analyses

- Cross-sectional, multi-level regression models of Round 2 audit data and contemporaneous admission rates.

- Difference-in-difference analyses comparing time trends in performance measures and admission rates between 2011/12 and 2013/14.

All analyses excluded tertiary centres.

Results In 2013/14, matched data were available on 8685 epilepsy-related admissions across 144 units.

In the longitudinal model, increased involvement of paediatric neurologists was associated with a small relative increase in admission rates over time $(\beta=0.13$ (95\% CI: 0.03 to 0.22$)$, $\mathrm{p}=0.007)$. There was no significant relationship between paediatric neurologist involvement and admission rates in the cross-sectional model $(\beta=-0.04(-0.14-0.05))$. No other perfomance indicator from the Epilepsy12 audit was associated with adjusted, unit-level admission rates on either cross-sectional or longitudinal models.

Conclusions Neither cross-sectional nor longitudinal models showed any consistent relationship between performance on the national clinical audit and unit-level admission rates for epilepsy among children and young people. This may reflect inconsistency in how the performance indicators are interpreted and measured, as well as the limitations of admission rates as a proxy for good seizure and quality of life among children and young people with epilepsy.

\section{G306 TRANSITIONAL CARE FROM PAEDIATRIC TO ADULT FOR ADOLESCENT WITH EPILEPSY: LOCAL SURVEY}

Y Teh, C Kallappa, M Awadh, M Khan. Paediatric, Heart of England, Birmingham, UK

10.1136/archdischild-2018-rcpch.298

Background Transition is a 'planned, purposeful movement of the young person from a child centred to an adult orientated healthcare system'. ${ }^{1}$ The transitional period from paediatric to adult care can be challenging for adolescence with epilepsy. Children with chronic illnesses often struggle more than their peers during adolescence, when healthcare providers change from the nurturing, family-centred style of paediatrics to the individualistic, 'autonomous' approach of adult healthcare. ${ }^{2}$ Poorly managed transitional period can therefore cause poor treatment compliance, and may have long term implications on the epileptic control and well-being of the adolescence. ${ }^{3}$ According to the National Institute for Health and Clinical Excellence (NICE), adolescence with epilepsy should have an agreed transition care period with joint adult and paediatric services. ${ }^{4}$ Hence, this qualitative survey aims to understand the patients' perspective of the joint transitional clinic.

Methods A 7 questions, qualitative questionnaire is used to survey the transitional of care for adolescence with epilepsy in joint adult and paediatric service in our trust. The clinic is held 4 times a year, and attended to $35-40$ patients with the presence of paediatrician and adult neurologist. The questionnaire was distributed to the adolescent/parents during the transitional clinic.

Results The survey showed that $90 \%$ of patients are satisfied with the care, with $60 \%$ of respondents suggested that more information on the cause of epilepsy and possible side effects of medication should be provided. The survey also highlighted the issues of lengthy clinic waiting times and the need for several clinic visits before transfer. Additionally, parental/patients' concerns around driving, career options, contraception, and epilepsy inheritance were also raised.

Conclusion There are no data available to which model would best fit the transitional epilepsy clinic. However, the survey found that patients/parents are generally satisfied with the transitional care of joint paediatric and adult services. The transition process is complex. Therefore, the utilisation of best available resources such as, epilepsy nurses, family support groups, specific interest groups, and primary care services should be planned early. Lastly, future survey should address the concerns on education about driving, career options, epilepsy inheritance and contraception in adolescence with epilepsy.

\section{REFERENCES}

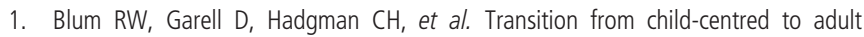
health-care systems for adolescents with chronic conditions: A position paper of the society for adolescent medicine. J Adol Health 1993;14:570-6.

2. Camfield P, Camfield C, Pohlmann-Eden B. Transition from paediatric to adult epilepsy care: A difficult process marked by medical and social crisis. Epilepsy Curr 2012 July-August; 12(Suppl 3):13-21.

3. Camfield P, Gibson P, Douglass L. Strategies for transitioning to adult care for youth with Lennox-Gastaut syndrome and related disorders. Epilepsia 2011;52 (Suppl 5):21-27.

4. National Institute of Clinical Excellence (NICE). Epilepsies: The diagnosis and management of the epilepsies in adults and children in primary and secondary care Clinical Guideline February 2016;137. NICE.

\section{G307(P) NATIONAL TRAINEES SERVICE EVALUATION OF THE MANAGEMENT OF IDIOPATHIC INTRACRANIAL HYPERTENSION (IIH) - PSEUDOTUMOR CEREBRI IN CHILDREN}

${ }^{1} \mathrm{~A}$ Amin, ${ }^{2} \mathrm{R}$ Spaull, ${ }^{1} \mathrm{R}$ Rattihalli, ${ }^{3} \mathrm{M}$ Atherton, ${ }^{4} \mathrm{~V}$ Mundada, ${ }^{5} \mathrm{E}$ Pilley, ${ }^{6} \mathrm{M}$ Moran, 'L Markham. 'University Hospitals Bristol, Bristol, UK; ${ }^{2}$ Gloucester Hospital, Gloucester, UK; ${ }^{3}$ Doncaster Hospital, Doncaster, UK; ${ }^{4}$ Royal London Hospital, London, UK; ${ }^{5}$ Edinburgh Hospital, Edinburgh, UK; ${ }^{6}$ Nottingham Hospital, Nottingham, UK

\subsection{6/archdischild-2018-rcpch.299}

Introduction IIH is characterised by raised intracranial pressure mostly affecting young females. It can lead to severe and irreversible visual loss. There is little consensus or evidence based 